\title{
Case report of domiciliary treatment with Tolvaptan in patients with advanced heart failure and persistent hyponatremia
}

\author{
Alberto Esteban-Fernández*, Nahikari Salterain-Gonzalez and Juan J Gavira-Gómez \\ Heart Failure Unit, Department of Cardiology, Clinica University of Navarra, Pamplona, Spain
}

\begin{abstract}
Management of patients with advanced heart failure and persistent hyponatremia remains a difficult challenge today. Classical treatment is not always enough, not only because of diuretics resistance, but also because of the natural course of heart failure and several comorbidities. Tolvaptan, a V2 receptor inhibitor in the kidneys, has demonstrated its utility and safety in refractory hyponatremia correction in patients with decompensated heart failure, but there is little experience about its use in management of chronic hyponatremia. We present a report of a case series with long term domiciliary treatment with Tolvaptan followed up in a Heart Failure unit.
\end{abstract}

\section{Introduction}

Hyponatremia, defined as plasmatic sodium $(\mathrm{Na})$ level lower than $135 \mathrm{mEq} / \mathrm{L}$, is known to be a marker of bad prognosis in patients with Heart Failure (HF) [1]. Until now, its treatment included water restriction, hypertonic fluids and diuretics, although there is not enough evidence about its effectiveness. Tolvaptan, an inhibitor of V2 receptor in the renal tubule, has been recently included in ESC (2012) and AHA/ACC (2013) HF guidelines [2,3] as an alternative treatment of refractory hyponatremia in decompensated HF. So far there is little experience about its usefulness in persistent and domiciliary hyponatremia management.

We present our clinical experience in the utility and safety of domiciliary treatment with Tolvaptan in patients with advanced HF and persistent hyponatremia.

\section{Methods}

We considered patients which started ambulatory treatment with Tolvaptan after at least two admissions due to decompensated HF and hyponatremia treated with classical methods and Tolvaptan. Patients with advanced kidney or hepatic diseases were excluded.

Week Tolvaptan dose was adjusted periodically (every week during the first month and then monthly) according to blood analytic results: $\mathrm{Na}$, potassium $(\mathrm{K})$, Creatinine (Cr), glomerular filtration rate (MDRD), urea and, in some cases, blood and urine osmolarity.

Follow-up was done by two specialized nurses and medical staff in our HF unit. Weight, congestion signs, symptoms of HF, blood pressure, heart rate and adverse effects related with Tolvaptan (e.g. hepatic disease) [4] were assessed.

\section{Results}

We present 6 patients ( 5 woman) with a mean age of $71 \pm 19$ years old (Table 1). The mean follow-up was 17 months. Patients 2 to 6 died during the follow-up (all of them died due to cardiovascular reasons except patient 5 who died because of pulmonary sepsis. Patient 1 had to discontinue Tolvaptan due to economic reasons and is still alive.

Sodium levels, as well as $\mathrm{K}$ and MDRD, were stable during Tolvaptan treatment (Figure 1), although there were not statistically significant differences. Also, weigh was stable during the follow-up (Figure 1), without statistical differences. The number of admissions was slightly reduced, specially because of symptomatic hyponatremia, although there were no statistically significant differences (Table 1).

None of the patients reported important adverse effects related to Tolvaptan except thirst.

\section{Discussion}

Hyponatremia is one of the most common electrolytic disorders in HF, related not only to poor prognosis in these patients [1], but also with unsteadiness, falls, and attention deficits specially in elder people [5]. Its adequate management, even at home, remains an important issue.

After the publication of some studies, specially the EVEREST, in which the effectiveness of Tolvaptan in increasing Na levels and diuresis (without affecting other electrolytes or renal function) was proved [6], it has been included in current HF guidelines [1,2]. Moreover, because of its aquaretic effect, it could be an alternative to associate to loop diuretics to stabilize Na levels and congestion signs in HF patients.

In our patients, chronic treatment was started after at least two admissions with decompensated HF and hyponatremia treated with

Correspondence to: Alberto Esteban-Fernández, Heart Failure Unit, Department of Cardiology, Clinica University of Navarra, Avenida Pio XII 36, 31008, Pamplona, Spain; E-mail: athalbertus@gmail.com

Key words: tolvaptan, heart failure, hyponatremia, domiciliary treatment

Received: February 08, 2015; Accepted: March 03, 2015; Published: March 07, 2015 


\section{Na level (mEq/L)}

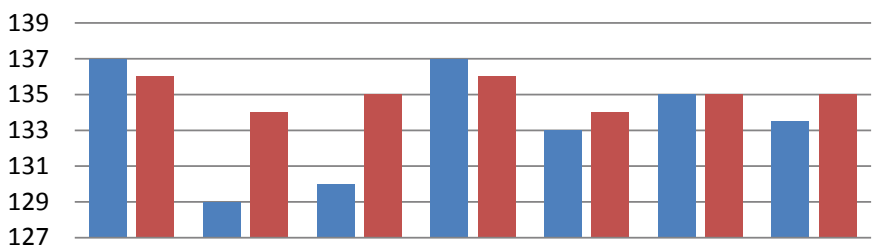

Patient 1 Patient 2 Patient 3 Patient 4 Patient 5 Patient 6 Mean

Basal nast follow-up

\section{K level (mEq/L)}

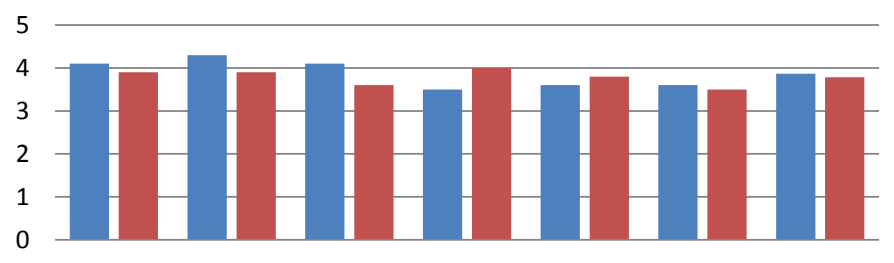

Patient 1 Patient 2 Patient 3 Patient 4 Patient 5 Patient 6 Mean

Basal Last follow-up

\section{$\operatorname{MDRD}\left(\mathrm{mL} / \mathrm{min} / 1.73 \mathrm{~m}^{2}\right)$}

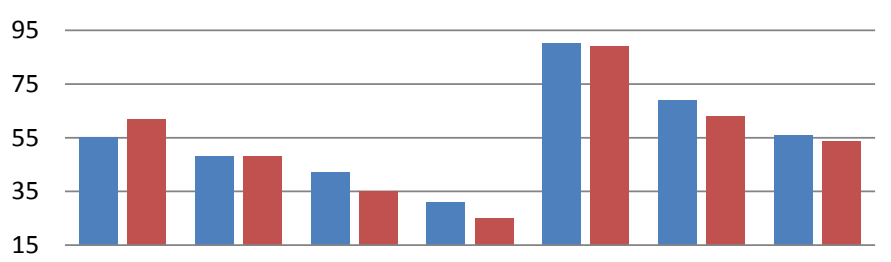

Patient 1 Patient 2 Patient 3 Patient 4 Patient 5 Patient 6 Mean

Basal Last follow-up

\section{Weight (kg)}

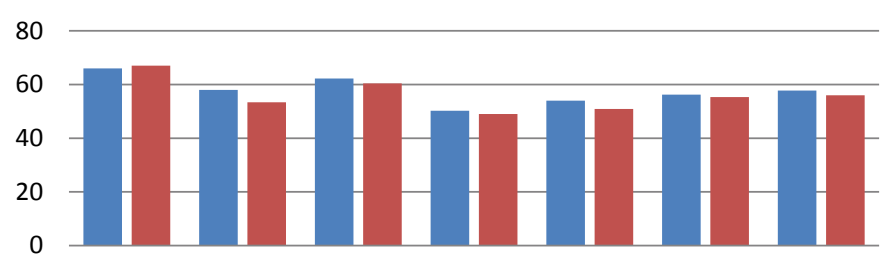

Patient 1 Patient 2 Patient 3 Patient 4 Patient 5 Patient 6 Mean

- Basal " Last follow-up

Figure 1. Evolution of analytical values (Na, K, MDRD) and weight in patients in chronic treatment with Tolvaptan during the follow-up.

standard management and Tolvaptan, in order to maintain or increase $\mathrm{Na}$ levels. In fact, we started it in two patients with normal Na but with past history of refractory hyponatremia controlled with Tolvaptan.

Sodium levels, through domiciliary treatment with Tolvaptan, were maintained stable as shown in Figure 1, although there were no statistically significant differences probably because of the small sample
Table 1. Basal characteristics and during the follow up of the patients in domiciliary treatment with Tolvaptan.

\begin{tabular}{|l|c|c|c|c|c|c|}
\hline & Patient & Patient & Patient & Patient & Patient & Patient \\
\hline Sex & Female & Female & Female & Female & Female & Male \\
\hline Age (years) & 91 & 63 & 81 & 77 & 37 & 74 \\
\hline Etiology & Hypert & Valv & Valv & Isch & Chemo & Isch+Valv \\
\hline LVSD (LVSF $<0.50)$ & No & No & No & Yes & No & Yes \\
\hline NYHA Class & III & II & I & II & II & II \\
\hline Systolic BP (mm Hg) & 125 & 110 & 110 & 140 & 98 & 95 \\
\hline DiastolicBP (mm Hg) & 60 & 70 & 60 & 60 & 72 & 50 \\
\hline Loop diuretics & Yes & Yes & Yes & Yes & Yes & Yes \\
\hline MRA & Yes & No & No & Yes & Yes & Yes \\
\hline ACE-I/ARB & Yes & No & No & No & No & No \\
\hline BB & No & No & No & Yes & Yes & Yes \\
\hline Tolvaptan Dose (mg) $*$ & 45 & 105 & 60 & 105 & 45 & 30 \\
\hline Follow-up (months) & 20 & 5 & 30 & 4 & 22 & 19 \\
\hline Admission for HF (no)** & $2 / 1$ & $2 / 1$ & $3 / 3$ & $2 / 1$ & $3 / 3$ & $1 / 2$ \\
\hline Admission for hypo*** (no) & $1 / 0$ & $0 / 0$ & $3 / 2$ & $0 / 0$ & $0 / 0$ & $0 / 0$ \\
\hline
\end{tabular}

Etiology denotes the cause of the myocardiopathy: valv is valvular, chemo is post chemotherapy, isch is ischaemic and Hypert is hypertensive. 1,3,6 and 12 make reference to the months of the follow up.

LVSD denotes left ventricular systolic dysfunction, MRA mineralcorticoid receptor antagonist, ACE-I angiotensin converting enzyme inhibitor, ARB angiotensin receptor antagonists, BB beta blockers.

*Week Tolvaptan dose at the beginning of the domiciliary treatment.

**Number of admission because of HF before and after treatment with Tolvaptan

**Number of admission because of symptomatic hyponatremia before and after treatment with Tolaptan

size. On the other hand, potassium, renal function and weight, did not worsen significantly during the follow-up (Figure 1). Therefore, Tolvaptan could be a good alternative added to chronic treatment of HF, specially loop diuretics, to maintain patient status, as reported in previous trials [7].

The only side effect linked to Tolvaptan was thirst, probably related to habitual water restriction in HF and other diuretics intake. There were not hepatic enzymes alterations or other side effects of the reported ones in the drug technical document to discontinue the treatment [4].

\section{Conclusion}

According to our results, domiciliary treatment with Tolvaptan, with HF nurses support, even in long term administration, is safe and helpful in chronic HF management. It could be considered in association with the standard treatment to maintain chronic status of these patients, specially in those with advanced HF, high diuretic requirements and persistent hyponatremia. Nevertheless, new trials should be performed to study the utility of Tolvaptan in this context.

\section{References}

1. Gheorghiade M, Abraham WT, Albert NM, GattisStough W, Greenberg BH, et al (2007) Relationship between admission serum sodium concentration and clinical outcomes in patients hospitalized for heart failure: an analysis from the OPTIMIZE-HF registry. Eur Heart J 28: 980-988. [Crossref]

2. McMurray JJ, Adamopoulos S, Anker SD, Auricchio A, Bohm M, et al. (2012) ESC Guidelines for the diagnosis and treatment of acute and chronic heart failure 2012: The Task Force for the Diagnosis and Treatment of Acute and Chronic Heart Failure 2012 of the European Society of Cardiology. Eur Heart J 33: 1787-847. [Crossref]

3. Yancy CW, Jessup M, Bozkurt B, Butler J, Casey DE Jr, et al. (2013) 2013 ACCF/ AHA guideline for the management of heart failure: a report of the American College 
Esteban-Fernández A (2015) Case report of domiciliary treatment with Tolvaptan in patients with advanced heart failure and persistent hyponatremia

of Cardiology Foundation/American Heart Association Task Force on Practice Guidelines. Circulation 62: e147-239. [Crossref]

4. European Medicines Agency (2009) CHMP assessment report for Samsca.

5. Renneboog B, Musch W, Vandemergel X, Manto MU, Decaux G (2006) Mild chronic hyponatremia is associated with falls, unsteadiness, and attention deficits. $\mathrm{Am} \mathrm{J} \mathrm{Med}$ 119: e1-8. [Crossref]
6. Gheorghiade M, Niazi I, Ouyang J, Czerwiec F, Kambayashi J, et al. (2003) Vasopressin V2 receptor blockade with tolvaptan in patients with chronic heart failure: results from a double-blind, randomized trial. Circulation 107: 2690-2696. [Crossref]

7. Berl T, Quittnat-Pelletier F, Verbalis JG, Schrier RW, Bichet DG, et al. (2010) Tolvaptan is safe and effective in chronic hyponatremia. J Am Soc Nephrol 21: 705712. [Crossref]

Copyright: (C2015 Esteban-Fernández A. This is an open-access article distributed under the terms of the Creative Commons Attribution License, which permits unrestricted use, distribution, and reproduction in any medium, provided the original author and source are credited. 